\title{
A Rare Presentation of Hepatic Hydrothorax in a Patient with Alcohol induced Liver Cirrhosis
}

\author{
Ganesh Kasinathan ${ }^{1, *}$, Naganathan Pillai ${ }^{2}$ \\ ${ }^{1}$ Department of Internal Medicine, Segamat Hospital, KM 6 Jalan Genuang, 85000 Segamat, Johor, Malaysia \\ ${ }^{2}$ Department of Internal Medicine, Monash University Malaysia, Bandar Sunway, Malaysia \\ *Corresponding author: ganeshkasinathan11@hotmail.com
}

\begin{abstract}
Hepatic hydrothorax is defined as significant pleural effusion greater than $500 \mathrm{ml}$ in a patient with liver cirrhosis without any underlying pulmonary, cardiac and pleural disease. This case report describes a 47 year old Indian gentleman who was diagnosed as alcohol induced liver cirrhosis, Child-Turcotte-Pugh score B, with gross ascites. He presented with recurrent right sided pleural effusion. Pleural fluid analysis revealed transudative pleural effusion. A diagnosis of hepatic hydrothorax was made after excluding other causes of pleural effusion. He did not respond to medical therapy and sodium restriction. His recurrent pleural effusion was treated with tube thoracostomy and chemical talc pleurodesis. He was referred to the tertiary hepatology unit for transjugular intrahepatic portosystemic shunt (TIPSS) and liver transplantation. Hepatic hydrothorax should always be suspected in a patient who presents with liver cirrhosis with portal hypertension and transudative pleural effusion.
\end{abstract}

Keywords: hepatic hydrothorax, cirrhosis, thoracostomy, transplantation, portal hypertension

Cite This Article: Ganesh Kasinathan, and Naganathan Pillai, "A Rare Presentation of Hepatic Hydrothorax in a Patient with Alcohol induced Liver Cirrhosis." American Journal of Medical Case Reports, vol. 5, no. 4 (2017): 104-106. doi: 10.12691/ajmcr-5-4-7.

\section{Introduction}

Hepatic hydrothorax is defined as transudative pleural effusion due to portal hypertension without any evidence of pulmonary, cardiac and pleural disease. The presence of portal hypertension but not end-stage liver disease is a requirement for the development of hepatic hydrothorax. [1] In most cases (85\%) hepatic hydrothorax develops on the right side, with $13 \%$ of cases occurring on the left side and $2 \%$ bilateral. [2] Ascites is usually present. Hepatic hydrothorax accounts for $2-3 \%$ of all pleural effusions. [3] Patients with ascites are able to tolerate a much greater amount of fluid amounting to 5-10 liters due to the large capacity of the peritoneal cavity. Those with pleural effusion are frequently symptomatic with fluid as little as 1-2 liters.

\section{Case Presentation}

A 47 year old Indian gentleman presented with worsening dyspnoea for the past 2 weeks associated with pleuritic chest pain. He denied any fever. No history of recent chest trauma or travel. His significant medical history includes alcohol induced liver cirrhosis with gross ascites and recurrent pleural effusion. His previous pleural effusion was treated with needle thoracocentesis. He was compliant to his diuretics and other medications. He works as a labourer with the local council. He has a long history of consumption of illicit liquor with high alcohol content. He is also a chronic smoker with no significant family history.

On examination, he was alert and well orientated in space and time. He had obvious abdominal distension with fluid thrill. Stigmata of chronic liver disease were present including bilateral palmar erythema, jaundice and gynaecomastia. His respiratory examination revealed reduced chest wall expansion, stony dullness and absent breath sounds over the entire right lung. There was bilateral pitting edema till the knees. The other examination systems were unremarkable. His blood pressure was $120 / 60$ with a heart rate of 88 beats per minute. His Child-Turcotte-Pugh score was B.

An urgent chest radiograph (Figure 1) showed massive right pleural effusion. He was anaemic with a haemoglobin of $8.4 \mathrm{~g} / \mathrm{dL}$. The other blood parameters are as shown in Table 1. The ultrasound of the abdomen showed an enlarged liver with coarse echotexture, splenomegaly and gross ascites.Abdominal paracentesis and thoracocentesis were performed which revealed transudative ascites and pleural effusion. The pleural fluid analysis is as shown in Table 2 which was similar in composition to his ascites. A CECT of the thorax showed right pleural effusion with no obvious lung or pleural mass. A 2D- transthoracic echocardiogram depicted a left ventricular ejection fraction of $65 \%$ with normal chamber sizes.

His medications were further optimized and he was placed on a strict sodium restriction diet. He was on oral furosemide, spironolactone, propranolol, omeprazole and folate. A chemical talc plurodesis was performed via tube thoracostomy in the ward. He was referred to the tertiary 
hepatology unit for transjugular intrahepatic portosystemic shunt (TIPSS) and liver transplantation.

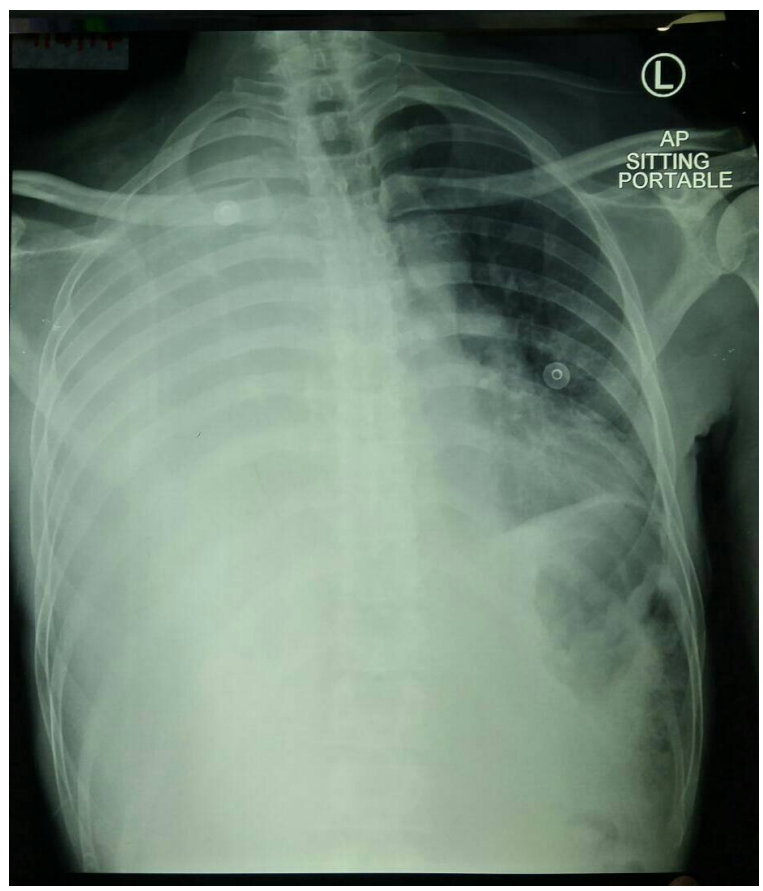

Figure 1. The chest radiograph shows massive right pleural effusion with mediastinal shift.

Table 1. Laboratory Values

\begin{tabular}{|l|c|}
\hline Variables (unit) & Value (Normal Range) \\
\hline Haemoglobin (g/dL) & $8.4(12-16)$ \\
\hline Mean Corpuscular Volume (fL) & $110(80-96)$ \\
\hline Total White Cell Count $\left(\right.$ per $\left.\mathrm{mm}^{3}\right)$ & $11900(4000-12000)$ \\
\hline Platelet $\left(\right.$ per $\left.\mathrm{mm}^{3}\right)$ & $78000(150000-400000)$ \\
\hline Urea $(\mathrm{mmol} / \mathrm{l})$ & $2.7(2.6-8.0)$ \\
\hline Sodium $(\mathrm{mmol} / \mathrm{l})$ & $128(136-145)$ \\
\hline Potassium $(\mathrm{mmol} / \mathrm{l})$ & $3.9(3.1-5.0)$ \\
\hline Creatinine $(\mathrm{umol} / \mathrm{l})$ & $51(40-80)$ \\
\hline Total Protein $(\mathrm{g} / \mathrm{l})$ & $62(60-80)$ \\
\hline Albumin $(\mathrm{g} / \mathrm{l})$ & $18(39.7-49.4)$ \\
\hline Aspartate Aminotransferase (U/L) & $42(0-45)$ \\
\hline Alanine Aminotransferase (U/L) & $46(0-41)$ \\
\hline Alkaline Phosphatase (U/L) & $193(40-129)$ \\
\hline Lactate Dehydrogenase (LDH) & $450(135-214)$ \\
\hline Total Bilirubin (umol/1) & $88(0-21)$ \\
\hline INR & $2.4(0.8-1.2)$ \\
\hline Random blood glucose (mmol) & $5.8(<7.8)$ \\
\hline Anti-HIV 1,2 & Not reactive \\
\hline HepBsAg & Not reactive \\
\hline Anti-Hep C & Not reactive \\
\hline Antinuclear Antibody & Not detected \\
\hline
\end{tabular}

Table 2. Pleural Fluid Values

\begin{tabular}{|l|c|}
\hline Plural fluid parameters (unit) & Value (normal range) \\
\hline Appearance & Straw coloured \\
\hline $\mathrm{pH}$ & $7.5(7.6-7.64)$ \\
\hline Total Protein $(\mathrm{g} / \mathrm{l})$ & $18.70(10-20)$ \\
\hline Glucose (mmol) & 5.5 \\
\hline Lactate Dehydrogenase (U/L) & 124 \\
\hline Gram stain & No organism seen \\
\hline Cell count & A few white blood cells seen \\
\hline Culture and sensitivity & No growth \\
\hline
\end{tabular}

\section{Discussion}

Portal hypertension is usually responsible for the formation of gross ascites in cirrhotic patients. The underlying pathogenesis leading to hepatic hydrothorax is similar to other causes of fluid accumulation in liver cirrhosis. Several mechanisms have been postulated to explain the incidence of hepatic hydrothorax in patients with liver cirrhosis and portal hypertension. These include the transfer of the peritoneal fluid into the pleural space via diaphragmatic defects, hypoalbuminemia resulting in decreased colloid osmotic pressure and lymphatic leakage from the thoracic duct. [4] The most common explainable mechanism is the passage of ascites from the peritoneal to the pleural cavity through diaphragmatic defects. These are typically less than $1 \mathrm{~cm}$ and may be microscopic and are generally located in the tendinous portion of the diaphragm. [5] Discontinuities in the collagen bundles that form the tendinous portion of the diaphragm contribute to this mechanism. The negative intrapleural pressure compared to that of the peritoneal cavity facilitates the one-way transfer of fluid and its subsequent trapping into the pleural space. [6] Herniation of the peritoneum into the pleural space also known as pleuroperitoneal blebs can develop because of the increase in gaps between the muscle fibers of the diaphragm with increased intra-abdominal pressure. [4] This theory is further supported by the fact that air, dyes, or radiolabeled substances intra-abdominally injected in patients with hepatic hydrothorax move rapidly into the pleural cavity. [7] The diaphragmatic defects resulting in hepatic hydrothorax are classified into four morphological types [8]:

- Type 1: no obvious defect

- Type 2: blebs lying in the diaphragm

- Type 3: fenestrations in the diaphragm

- Type 4: multiple gaps in the diaphragm

Most of the defects tend to occur on the right diaphragm due to the anatomical proximity of the liver.

Treatment for hepatic hydrothorax involves reducing the portal hypertension which is responsible for ascites formation, preventing escape of fluid via diaphragmatic defects and draining of pleural fluid. Medical management and nutritional recommendation is mainly concerning sodium diet restriction and enhancing renal sodium excretion via the combination use of diuretics such as furosemide and spironolactone. Some patients fail to respond to medical management resulting in refractory hepatic hydrothorax. Liver transplantation is the best option for these patients. [9] Transjugular intrahepatic portosystemic shunt (TIPSS) may lead to a symptomatic improvement in $70-80 \%$ of patients with refractory hydrothorax. [10] The common complications associated with transjugular intrahepatic portosystemic shunt (TIPSS) are precipitation of hepatic encephalopathy and shunt occlusion. In carefully selected patients with MELD (Model for End stage Liver disease) score $<15$, CTP (Child-Turcotte-Pugh) A or B and age $<60$ years, transjugular intrahepatic portosystemic shunt (TIPSS) can be an effective treatment of refractory hydrothorax and can be used as a bridge to liver transplantation. [4]

Chemical pleurodesis via tube thoracostomy is commonly used to treat patients with recurrent malignant 
based pleural effusions. Experience suggests that hepatic hydrothorax is the most difficult type of nonmalignant based pleural effusion to treat with chemical pleurodesis. Because of the rapid migration of fluid from the abdomen into the pleural space, it is often difficult to keep the two pleural surfaces apposed long enough for the inflammatory process to result in pleural symphysis. [11] Other methods, such as video-assisted thoracoscopy with pleurodesis have been reported with more encouraging results. [12] Surgical repair of diaphragmatic defects serves another option of treatment for refractory hepatic hydrothorax. In a study involving eight patients with refractory hepatic hydrothorax, video assisted thoracoscopy was used to repair diaphragmatic defects in addition to pleurodesis. [13].

\section{Conclusion}

Hepatic hydrothorax should always be suspected in a patient who presents with liver cirrhosis with portal hypertension and transudative pleural effusion. A diagnostic thoracocentesis should be performed in such a patient. Optimization of medical therapy and sodium restriction are important in non-refractory hepatic hydrothorax. Those with recurrent hepatic hydrothorax may benefit from other therapeutic modalities including liver transplantation.

\section{Acknowledgements}

None

\section{Statement of Competing Interests}

None.

\section{List of Abbreviations}

None.

\section{References}

[1] Karen LK, Cardenas A. Hepatic hydrothorax. Semin Respir Crit Care Med. 2012; 33: 3-10.

[2] Kinasewitz GT, Keddissi JI. Hepatic hydrothorax. Curr Opin Pulm Med. 2003; 9: 261-5.

[3] Esteve M, Xiol X, Fernadez F, Gonzalez F, Baliellas C: Treatment and outcome of hydrothorax in liver cirrhosis. J Clin Nutr Gastroenterol. 1986; 1: 139-144.

[4] Singh A, Bajwa A, Shujaat A. Evidence based review of the management of hepatic hydrothorax. Respiration 2013; 86: 155-173.

[5] Zenda T, Miyamoto S, Murata S, Mabuchi H. Detection of diaphragmatic defect as the cause of severe hepatic hydrothorax with magnetic resonance imaging. Am J Gastroenterol. 1998; 93: 2288 .

[6] Siddappa PK, Kar P. Hepatic Hydrothorax. Tropical Gastroenterology 2009; 30(3):135-141.

[7] Foschi F, Piscaglia F, Pompili M, Corbelli C, Marano G. Real-Time Contrast-Enhanced Ultrasound - a New Simple Tool for Detection of Peritoneal-Pleural Communications in Hepatic Hydrothorax. Ultraschall Med. 2008 Aug; 22: 18726840.

[8] Huang PM, Chang YL, Yang CY, Lee YC. The morphology of diaphragmatic defects in hepatic hydrothorax: thoracoscopic finding. J Thorac Cardiovasc Surg. 2005; 130: 141-145.

[9] Light RW, Macgregor MI, Luchsinger PC, Ball WC Jr. Pleural effusions: the diagnostic separation of transudates and exudates. Ann Intern Med 1972; 77: 507-513.

[10] Siegerstetter V, Deibert P, Ochs A, Olschewski M, Blum HE, Rossle M. Treatment of refractory hepatic hydrothorax with transjugular intrahepatic portosystemic shunt: long-term results in 40 patients. Eur J Gastroenterol Hepatol.. 2001; 13: 529-34.

[11] Falchuk KR, Jacoby I, Colucci WS, Rybak ME. Tetracyclineinduced pleural symphysis for recurrent hydrothorax complicating cirrhosis.A new approach to treatment. Gastroenterology. 1977; 72: 319 .

[12] Temes RT, Davis MS, Follis FM, Pett SB Jr, Wernly JA. Video thoracoscopic treatment of hepatic hydrothorax. Ann Thorac Surg. 1997; 64: 1468-9.

[13] Mouroux J, Perrin C, Venissac N, et al. Management of pleural effusion of cirrhotic origin. Chest 1996; 109:1093. 